\begin{tabular}{c} 
International Journal of Advanced Mathematical Sciences, 5(1)(2017) $20-26$ \\
SPC \\
Website: $\begin{array}{c}\text { www.sciencepubco.com/index.php/IJAMS } \\
\text { doi: } 10.14419 / \text { ijams. } v \text { Sil.7270 } \\
\text { Research paper }\end{array}$ \\
\hline
\end{tabular}

\title{
Algorithms of Common Solutions for a Fixed Point of Hemicontractive-type Mapping and a Generalized Equilibrium Problem
}

\author{
Habtu Zegeye $^{1^{*}}{\text {, Tesfalem Hadush } \text { Meche }^{2} \text { and Mengistu Goa Sangago }}^{3}$ \\ ${ }^{1}$ Department of Mathematics, College of Sciences, Botswana International University of Science and Technology, Private Mail Bag 16, \\ Palapye, Botswana \\ ${ }^{2}$ Department of Mathematics, College of Natural and Computational Sciences, Addis Ababa University, P.O.Box 1176, Addis Ababa, \\ Ethiopia \\ ${ }^{3}$ Department of Mathematics, College of Natural and Computational Sciences, Addis Ababa University, P.O.Box 31167, Addis Ababa, \\ Ethiopia \\ *Corresponding author E-mail: habtuzh@yahoo.com
}

\begin{abstract}
In this paper, we introduce and study an iterative algorithm for finding a common element of the set of fixed points of a Lipschitz hemicontractive-type multi-valued mapping and the set of solutions of a generalized equilibrium problem in the framework of Hilbert spaces. Our results improve and extend most of the results that have been proved previously by many authors in this research area.
\end{abstract}

Keywords: Fixed Points of Mappings; Generalized Equilibrium Problem; Hemicontractive-type Multi-valued Mapping; Iterative Algorithm; Strong Convergence.

\section{Introduction}

Let $H$ be a real Hilbert space with inner product $\langle.,$.$\rangle and norm \|$.$\| .$ Let $C$ be a nonempty subset of $H$.

A mapping $T: C \longrightarrow H$ is called $k$-strictly pseudocontractive if there exists a constant $k \in[0,1)$ such that

$$
\|T x-T y\|^{2} \leq\|x-y\|^{2}+k\|x-T x-(y-T y)\|^{2},
$$

for all $x, y \in C$. If $k=1$ in (1.1), then $T$ is called pseudocontractive mapping.

A mapping $T: C \rightarrow H$ is called Lipschitzian if there exists $L \geq 0$ such that $\|T x-T y\| \leq L\|x-y\|, \forall x, y \in C$. If $L=1$, then $T$ is called nonexpansive and if $L \in[0,1)$ then $T$ is called a contraction.

Observe that the class of pseudocontractive mappings includes the class of strictly pseudocontractive mappings and hence the class of nonexpansive and contraction mappings.

Let $C B(C)$ denote the family of nonempty, closed and bounded subsets of $C$. The Pompeiu-Hausdorff metric ([2]) on $C B(C)$ is defined by

$$
D(A, B)=\max \left\{\sup _{x \in A} d(x, B), \sup _{y \in B} d(y, A)\right\},
$$

for all $A, B \in C B(C)$, where $d(x, B)=\inf \{\|x-b\|: b \in B\}$.

A multi-valued mapping $T: C \longrightarrow C B(C)$ is said to be $k$-strictly pseudocontractive if there exists a constant $k \in[0,1)$ such that

$$
D^{2}(T x, T y) \leq\|x-y\|^{2}+k\|(x-u)-(y-v)\|^{2},
$$

for all $x, y \in C$ and $u \in T x, v \in T y$. If $k=1$ in (1.2), then $T$ is called pseudocontractive mapping.

A multi-valued mapping $T$ is called Lipschitzian if there exists $L \geq 0$ such that

$$
D(T x, T y) \leq L\|x-y\|, \forall x, y \in C .
$$

If $L=1$, then $T$ is called nonexpansive and if $L \in[0,1)$ then $T$ is called a contraction mapping.

An element $x \in C$ is called a fixed point of $T: C \longrightarrow C$ (resp., $T$ : $C \longrightarrow C B(C)$ ) if $x=T x$ (resp., $x \in T x$ ). The set of fixed points of $T$ is denoted by $F(T)$. We write $x_{n} \rightarrow x$ to indicate that the sequence $\left\{x_{n}\right\}$ converges weakly to $x$ and $x_{n} \rightarrow x$ to indicate that the sequence $\left\{x_{n}\right\}$ converges strongly to $x$.

A mapping $T: C \longrightarrow H$ is called quasi-nonexpansive if $F(T)$ is nonempty and the inequality

$$
\|T x-p\| \leq\|x-p\|
$$

holds for all $p \in F(T), x \in C$.

The mapping $T$ is called hemicontractive if $F(T)$ is nonempty and it satisfies:

$$
\|T x-p\|^{2} \leq\|x-p\|^{2}+\|x-T x\|^{2},
$$

for all $p \in F(T), x \in C$.

A multi-valued mapping $T: C \longrightarrow C B(C)$ is called quasinonexpansive if $F(T) \neq \emptyset$ and for all $p \in F(T), x \in C$, we have

$$
D(T x, T p) \leq\|x-p\| .
$$


A multivalued mapping $T: C \longrightarrow C B(C)$ is said to be hemicontractive-type if $F(T) \neq \emptyset$ and for all $p \in F(T), x \in C$,

$$
D^{2}(T x, T p) \leq\|x-p\|^{2}+\|x-u\|^{2}, \forall u \in T x .
$$

We observe that every nonexpansive mapping with $F(T) \neq \emptyset$ is quasi-nonexpansive mapping, and every pseudocontractive mapping $T$ with $F(T) \neq \emptyset$ and $T(p)=\{p\}, \forall p \in F(T)$ is hemicontractivetype mapping. It is also easy to see that every quasi-nonexpansive mapping is hemicontractive-type mapping. Next, we give an example of a hemicontractive-type mapping which is not quasi-nonexpansive and pseudocontractive mappings.

Example 1.1. Let $T: \mathbb{R} \longrightarrow C B(\mathbb{R})$ be given by

$$
T x=[-3 x,-2 x], \text { if } x \geq 0 \text { and } T x=[-2 x,-3 x], \text { if } x<0 .
$$

Then, zero is the only fixed point of $T$, that is, $F(T)=\{0\}$ and for all $x \in \mathbb{R} \backslash\{0\}$, we have

$$
\begin{aligned}
D(T x, T 0) & =\max \left\{\sup _{a \in T x} d(a, T 0), \sup _{b \in T 0} d(b, T x)\right\} \\
& =\max \left\{\sup _{a \in T x}|a|, d(0, T x)\right\} \\
& =\max \{3|x|, 2|x|)\}=3|x|>|x-0|,
\end{aligned}
$$

which shows that $T$ is not quasi-nonexpansive. And for every $x \in \mathbb{R}$,

$$
d(x, T x)=\inf _{a \in T x}|a-x|=|x+2 x|=3|x| .
$$

Thus, from (1.4), we have

$$
\begin{aligned}
D^{2}(T x, T 0) & =9|x|^{2}=|x-0|^{2}+8|x|^{2} \\
& \leq|x-0|^{2}+9|x|^{2}=|x-0|^{2}+(d(x, T x))^{2} \\
& \leq|x-0|^{2}+|x-u|^{2}, \text { for all } u \in T x .
\end{aligned}
$$

This shows that $T$ is hemicontractive-type mapping. However, it is not a pseudocontractive. Indeed, for $x=1, y=2, u=-3 \in T x$ and $v=-4 \in T y$, we have

$$
D^{2}(T x, T y)=9>1+4=|x-y|^{2}+|x-u-(y-v)|^{2} .
$$

Therefore, the class of multi-valued hemicontractive-type mappings is more general in the sense that it includes properly the class of multi-valued quasi-nonexpansive mappings and the class of pseudocontractive mappings $T$ with $F(T) \neq \emptyset$ and $T(p)=\{p\}, \forall p \in F(T)$.

Let $T: C \longrightarrow C B(C)$ be a multi-valued mapping, $I-T$ (where $I$ is the identity mapping on $C$ ) is said to be demiclosed at zero if $\left\{x_{n}\right\} \subset C$ such that $x_{n} \rightarrow x$ and $\lim _{n \rightarrow \infty} d\left(x_{n}, T x_{n}\right)=0$ implies $x \in T x$.

Several authors have studied iterative algorithms for approximating fixed points of various classes of nonlinear mappings (including hemicontractive mapping); see, for example, $[5,8,14,15,21]$ and the references cited therein. In 2007, Rafiq [13] introduced the following Mann-type implicit iteration process and showed that the process converges strongly to a fixed point of a continuous hemicontractive mapping $T$ from a compact and convex subset $C$ of a real Hilbert space into itself:

$$
\left\{\begin{array}{l}
x_{0} \in C \\
x_{n}=\alpha_{n} x_{n-1}+\left(1-\alpha_{n}\right) T x_{n}, \quad \forall n \geq 1
\end{array}\right.
$$

where $\left\{\alpha_{n}\right\} \subset[0,1]$ satisfying some appropriate conditions.

Recently, Woldeamanuel et al.[19] extended the results of Rafiq [13] from single-valued hemicontractive to multi-valued hemicontractivetype mapping. More precisely, they studied the following three-step iterative algorithm for finding a common point of fixed points of a finite family of Lipschitz and hemicontractive-type multi-valued mappings under mild assumption on the parameters:

$$
\left\{\begin{array}{l}
z_{n}=\left(1-\gamma_{n}\right) x_{n}+\gamma_{n} w_{n}, \quad w_{n} \in T_{n} y_{n}, \\
y_{n}=\left(1-\beta_{n}\right) x_{n}+\beta_{n} u_{n}, \quad u_{n} \in T_{n} x_{n}, \\
x_{n+1}=\alpha_{n} w+\left(1-\alpha_{n}\right) z_{n}, \quad \forall n \geq 1 .
\end{array}\right.
$$

They proved that the sequence $\left\{x_{n}\right\}$, generated by (1.5), converges strongly to some point $p$ in $\cap_{i=1}^{N} F\left(T_{i}\right)$ nearest to $w$.

A mapping $A: C \longrightarrow H$ is called $\alpha$-inverse strongly monotone if there exists a positive real number $\alpha$ such that

$$
\langle A x-A y, x-y\rangle \geq \alpha\|A x-A y\|^{2}, \forall x, y \in C .
$$

Note that every $\alpha$-inverse strongly monotone mapping is $\frac{1}{\alpha}$-Lipschitz mapping. However, the converse may not hold. For example, the mapping $A:(0,1) \longrightarrow \mathbb{R}$ defined by $A x=-x$ is Lipschitz but not $\alpha$-inverse strongly monotone.

A mapping $A: C \longrightarrow H$ is called monotone if

$$
\langle A x-A y, x-y\rangle \geq 0, \forall x, y \in C .
$$

Clearly, the class of monotone mappings includes the class of $\alpha$ inverse strongly monotone and the inclusion is proper. To see this, consider the mapping $A:(0,1) \longrightarrow \mathbb{R}$ given by $A x=-\frac{1}{x}$. Then, for every $x, y \in C$, we have

$$
\langle x-y, A x-A y\rangle=\left\langle x-y,-\frac{1}{x}+\frac{1}{y}\right\rangle=\frac{1}{x y}\|x-y\|^{2} \geq 0,
$$

which shows that $A$ is monotone mapping. However, since $|A x-A y|=\left|\frac{-1}{x}+\frac{1}{y}\right|=\frac{1}{x y}|x-y|$ and $\frac{1}{x y} \rightarrow \infty$ as $x y \rightarrow 0$, then we can not find a constant $L \geq 0$ such that $|A x-A y| \leq L|x-y|$, for all $x, y \in(0,1)$. Hence, $A$ is not Lipschitz and so it is not $\alpha$-inverse strongly monotone mapping.

Let $C$ be a nonempty, closed and convex subset of a real Hilbert space $H$. Let $F: C \times C \longrightarrow \mathbb{R}$ be a bifunction and $A: C \longrightarrow H$ be a nonlinear mapping. The generalized equilibrium problem is to find $z \in C$ such that

$$
F(z, y)+\langle A z, y-z\rangle \geq 0, \quad \forall y \in C .
$$

In this paper, the set of solutions of problem (1.6) is denoted by $E P(F, A)$, i.e.,

$$
E P(F, A)=\{z \in C: F(z, y)+\langle A z, y-z\rangle \geq 0, \forall y \in C\} .
$$

In the case of $A \equiv 0$, then the problem (1.6) reduces to the equilibrium problem:

$$
\text { Finding } z \in C \text { such that } F(z, y) \geq 0, \forall y \in C \text {. }
$$

The set of solutions of problem (1.7) is denoted by $E P(F)$. In the case of $F \equiv 0$, then the problem (1.6) reduced to finding $z \in C$ such that

$$
\langle A z, y-z\rangle \geq 0, \forall y \in C,
$$

which is called the classical variational inequality problem. The set of solutions of problem $(1.8)$ is denoted by $\operatorname{VI}(C, A)$.

Clearly, if a point $z \in V I(C, A) \cap E P(F)$, then $z \in E P(F, A)$, however, the converse may not hold as we can see from the following example.

Example 1.2. Let $H=\mathbb{R}$ and $C=[0,1]$. Define $F: C \times C \rightarrow \mathbb{R}$ by $F(x, y)=x+y$ and $A: C \rightarrow H$ by $A x=\frac{x}{2}$. Then, for $x=1$, we have

$$
F(x, y)+\langle A x, y-x\rangle=(1+y)+\frac{1}{2}(y-1)=\frac{1}{2}(3 y+1) \geq 0,
$$

for all $y \in C$, which implies that $1 \in E P(F, A)$. But, since $\langle A 1, y-$ $1\rangle=\frac{1}{2}(y-1) \leq 0$ for all $y \in C$, then $1 \notin V I(C, A)$ and hence $1 \notin$ $E P(F) \cap V I(C, A)$. 
Assumption 1.3. Let $C$ be a nonempty, closed and convex subset of a real Hilbert space $H$. In the sequel, let $F$ be a bifunction of $C \times C$ into $\mathbb{R}$ satisfying the following conditions:

(A1) $F(x, x)=0, \forall x \in C$;

(A2) $F$ is monotone, i.e., $F(x, y)+F(y, x) \leq 0, \forall x, y \in C$;

(A3) $\lim _{t \downarrow 0} F(t z+(1-t) x, y) \leq F(x, y), \forall x, y, z \in C$;

(A4) For each $x \in C, y \mapsto F(x, y)$ is convex and lower semicontinuous.

Remark 1.4. Let $C$ be a nonempty, closed and convex subset of a real Hilbert space $H$. Let $F$ be a bifunction from $C \times C$ into $\mathbb{R}$ satisfying Assumption 1.3 and let $A$ be a continuous monotone mapping. Define $G: C \times C \longrightarrow \mathbb{R}$ by

$$
G(x, y)=F(x, y)+\langle A x, y-x\rangle,
$$

then it is easy to see that the bifunction G satisfies Assumption 1.3. Thus, the generalized equilibrium problem (1.6) is equivalent to the following equilibrium problem:

Find $z \in C$ such that $G(z, w) \geq 0$, for all $w \in C$.

Generalized equilibrium problem is more general in the sense that it includes equilibrium problems and hence variational inequalitiy, optimization problem, Nash equilibrium problem etc. Consequently, many authors have shown their interest in constructing an iterative algorithm for approximating common solution of generalized equilibrium and fixed point problems. We describe some of them as follows:

In 2008, Moudafi [11] introduced an iterative scheme for finding a common element of the set of solutions of generalized equilibrium problems and the set of fixed points of nonexpansive single-valued mapping in a Hilbert space setting and then obtained the following weak convergence theorem.

Theorem 1.5. [11] Let $C$ be a nonempty, closed and convex subset of a real Hilbert space $H$ and $F: C \times C \longrightarrow \mathbb{R}$ be a bifunction satisfying Assumption 1.3. Let $A$ be an $\alpha$-inverse strongly monotone mapping of $C$ into $H$, and let $T$ be a nonexpansive mapping of $C$ in to itself such that $F(T) \cap E P(F, A)$ is nonempty. Let $x_{0} \in C$ and $\left\{y_{n}\right\},\left\{x_{n}\right\}$ be sequences generated by

$$
\left\{\begin{array}{l}
F\left(y_{n}, y\right)+\left\langle A x_{n}, y-y_{n}\right\rangle \\
+\frac{1}{r_{n}}\left\langle y-y_{n}, y_{n}-x_{n}\right\rangle \geq 0, \forall y \in C \\
x_{n+1}=\alpha_{n} x_{n}+\left(1-\alpha_{n}\right) T y_{n}, \forall n \geq 0,
\end{array}\right.
$$

where $\left\{r_{n}\right\} \subset[a, b]$ for some $a, b \in(0,2 \alpha)$ and $\left\{\alpha_{n}\right\} \subset[c, d]$ for some $c, d \in(0,1)$. Then, $\left\{x_{n}\right\}$ converges weakly to $z \in F(T) \cap$ $E P(F, A)$, where $z=\lim _{n \rightarrow \infty} P_{F(T) \cap E P(F, A)} x_{n}$.

In [17], motivated by the result given by Moudafi [11], Takahashi and Takahashi proposed an iterative scheme and proved the following convergence theorem.

Theorem 1.6. [17] Let $C$ be a nonempty, closed and convex subset of a real Hilbert space $H$ and $F: C \times C \longrightarrow \mathbb{R}$ be a bifunction satisfying Assumption 1.3. Let $A$ be an $\alpha$-inverse strongly monotone mapping of $C$ into $H$ and $T: C \longrightarrow C$ be a nonexpansive mapping such that $F(T) \cap E P(F, A)$ is nonempty. Let $u, x_{1} \in C$ and $\left\{z_{n}\right\},\left\{x_{n}\right\}$ be sequences generated by

$$
\left\{\begin{array}{l}
F\left(z_{n}, y\right)+\left\langle A x_{n}, y-z_{n}\right\rangle \\
+\frac{1}{\lambda_{n}}\left\langle y-z_{n}, z_{n}-x_{n}\right\rangle \geq 0, \forall y \in C \\
y_{n}=\alpha_{n} u+\left(1-\alpha_{n}\right) z_{n} \\
x_{n+1}=\beta_{n} x_{n}+\left(1-\beta_{n}\right) T y_{n}, \forall n \geq 1,
\end{array}\right.
$$

where $\left\{\alpha_{n}\right\} \subset[0,1],\left\{\beta_{n}\right\} \subset[0,1]$ and $\left\{\lambda_{n}\right\} \subset[0,2 \alpha]$ satisfying:

i. $0<a \leq \lambda_{n} \leq b<2 \alpha, 0<c \leq \beta_{n} \leq d<1$;

ii. $\lim _{n \rightarrow \infty}\left|\lambda_{n}-\lambda_{n+1}\right|=0, \lim _{n \rightarrow \infty} \alpha_{n}=0$ and $\sum_{n=1}^{\infty} \alpha_{n}=\infty$.
Then, $\left\{x_{n}\right\}$ converges strongly to $z_{0}=P_{F(T) \cap E P(F, A)}(u)$.

Recently, Wong et al.[4] introduced the following implicit viscosity approximation method, starting from $x_{0} \in C$, for finding common point of the set of solutions of generalized equilibrium problem (1.6) and the set of fixed points of a continuous pseudocontractive mapping in Hilbert spaces:

$$
\left\{\begin{array}{l}
F\left(z_{n-1}, y\right)+\left\langle A x_{n-1}, y-z_{n-1}\right\rangle \\
+\frac{1}{\lambda_{n}}\left\langle y-z_{n-1}, z_{n-1}-x_{n-1}\right\rangle \geq 0, \forall y \in C \\
y_{n}=\beta_{n} f\left(x_{n-1}\right)+\gamma_{n} S z_{n-1}+\left(1-\beta_{n}-\gamma_{n}\right) x_{n-1} \\
x_{n}=\alpha_{n} y_{n}+\left(1-\alpha_{n}\right) T x_{n}, \forall n \in \mathbb{N}
\end{array}\right.
$$

where $F: C \times C \longrightarrow \mathbb{R}$ is a bifunction, $A: C \longrightarrow H$ is an $\alpha$-inverse strongly monotone mapping, $f: C \longrightarrow C$ is a fixed contraction mapping, $S: C \longrightarrow C$ is a nonexpansive mapping, and $T: C \longrightarrow C$ is a continuous pseudocontractive mapping such that $F(T) \cap E P(F, A)$ is nonempty. They proved that the sequence $\left\{x_{n}\right\}$, generated by (1.9), converges strongly to a point in $F(T) \cap E P(F, A)$ provided that the sequences $\left\{\alpha_{n}\right\},\left\{\beta_{n}\right\},\left\{\gamma_{n}\right\} \subset(0,1]$ and $\left\{\lambda_{n}\right\} \subset(0,2 \alpha]$ satisfy some appropriate control conditions.

In this paper, motivated and inspired by the idea of Iiduka and Takahashi [7], Takahashi and Takahashi [16, 17], Wong et al.[4], we introduce an iterative algorithm for finding a common element of the solution set of a generalized equilibrium problem (1.6) and the set of fixed points of a multi-valved Lipschitz hemicontractive-type mapping. We also establish a strong convergence theorem in the framework of Hilbert spaces. The results presented in this paper improve and extend the corresponding results announced by Meche et al.[10], Moudafi [11], Takahashi and Takahashi [16, 17], Wong et.al.[4] and some other results that have been obtained previously in this research area.

\section{Preliminaries}

Throughout this section unless otherwise stated, let $C$ be a nonempty, closed and convex subset of a real Hilbert space $H$. For every point $x \in H$, there exists a unique nearest point in $C$, denoted by $P_{C} x$, such that

$$
\left\|x-P_{C} x\right\|=\inf \{\|x-y\|: y \in C\} .
$$

$P_{C}$ is called the metric projection of $H$ onto $C$. We note that the metric projection $P_{C}$ is a nonexpansive mapping from $H$ onto $C$. It also satisfies the following property: for any $x \in H$ and $z \in C$,

$$
z=P_{C} x \text { if and only if }\langle x-z, z-y\rangle \geq 0 \text {, for all } y \in C .
$$

Furthermore, for any $x, y \in H$, we have

$$
\|x-y\|^{2}=\|x\|^{2}+\|y\|^{2}-2\langle x, y\rangle .
$$

We note that $(I-T)$ is demiclosed at zero whenever $T: C \longrightarrow C$ is a nonexpansive mapping, (see [1]).

We need the following lemmas for the proof of our main results.

Lemma 2.1. [22] Let $H$ be a real Hilbert space. Then for all $x_{i} \in H$ and $\alpha_{i} \in[0,1]$ for $i=1,2, \cdots, n$ such that $\alpha_{1}+\alpha_{2}+\cdots+\alpha_{n}=1$ the following equality holds:

$\left\|\alpha_{1} x_{1}+\alpha_{2} x_{2}+\cdots+\alpha_{n} x_{n}\right\|^{2}=\sum_{i=1}^{n} \alpha_{i}\left\|x_{i}\right\|^{2}-\sum_{1 \leq i, j \leq n} \alpha_{i} \alpha_{j}\left\|x_{i}-x_{j}\right\|^{2}$.

Lemma 2.2. Let $H$ be a real Hilbert space. Then, for any given $x, y \in H$, we have the following inequality:

$$
\|x+y\|^{2} \leq\|x\|^{2}+2\langle y, x+y\rangle .
$$


Lemma 2.3. [3, 6] Let $F$ be a bifunction from $C \times C$ into $\mathbb{R}$ satisfying Assumption 1.3. For $r>0$ and for all $x \in H$, define a mapping $T_{r}: H \longrightarrow C$ as follows:

$$
T_{r} x=\left\{z \in C: F(z, y)+\frac{1}{r}\langle y-z, z-x\rangle \geq 0, \forall y \in C\right\} .
$$

Then, the following hold:

(1) $T_{r}$ is nonempty and single valued;

(2) $T_{r}$ is firmly nonexpansive, i.e., $\left\|T_{r} x-T_{r} y\right\|^{2} \leq\left\langle T_{r} x-T_{r} y, x-y\right\rangle$, $\forall x, y \in H$

(3) $F\left(T_{r}\right)=E P(F)$;

(4) $E P(F)$ is closed and convex.

From Lemma 2.3 we observe that for each given $x \in H$, there exists a unique element $z \in C$ such that $z=T_{r} x$.

Lemma 2.4. [12] Let $H$ be a Hilbert space. Let $A, B \in C B(H)$ and $a \in A$. Then, for $\varepsilon>0$, there exists a point $b \in B$ such that $\|a-b\| \leq D(A, B)+\varepsilon$. As a consequence of this, taking $\varepsilon=D(A, B)$, we obtain that $\|a-b\| \leq 2 D(A, B)$.

Lemma 2.5. [20] Let $\left\{b_{n}\right\}$ be a sequence of nonnegative real numbers satisfying the following relation:

$$
b_{n+1} \leq\left(1-\alpha_{n}\right) b_{n}+\alpha_{n} \delta_{n}, \text { for } n \geq n_{0},
$$

where $\left\{\alpha_{n}\right\} \subset(0,1)$ and $\delta_{n} \subset \mathbb{R}$ satisfying the following conditions:

$$
\lim _{n \rightarrow \infty} \alpha_{n}=0, \sum_{n=1}^{\infty} \alpha_{n}=\infty, \text { and } \limsup _{n \rightarrow \infty} \delta_{n} \leq 0 .
$$

Then, $\lim _{n \rightarrow \infty} b_{n}=0$.

Lemma 2.6. [9] Let $\left\{a_{n}\right\}$ be a sequence of real numbers such that there exist a subsequence $\left\{n_{i}\right\}$ of $\{n\}$ such that $a_{n_{i}}<a_{n_{i}+1}$, for all $i \in \mathbb{N}$. Then there exists a nondecreasing sequence $\left\{m_{k}\right\} \subset \mathbb{N}$ such that $m_{k} \rightarrow \infty$ and the following properties are satisfied by all (sufficiently large) numbers $k \in \mathbb{N}$ :

$$
a_{m_{k}} \leq a_{m_{k}+1} \text { and } a_{k} \leq a_{m_{k}+1} .
$$

In fact, $m_{k}=\max \left\{j \leq k: a_{j} \leq a_{j+1}\right\}$.

\section{Main Result}

In this section, we prove strong convergence theorems for a generalized equilibrium problem and a fixed point problem for a multivalued Lipschitz hemicontractive-type mapping.

Theorem 3.1. Let $C$ be a nonempty, closed and convex subset of a real Hilbert space $H$. Let $A: C \longrightarrow H$ be a continuous monotone mapping and $F: C \times C \longrightarrow \mathbb{R}$ be a bifunction satisfying Assumption 1.3. Let $T: C \longrightarrow C B(C)$ be a Lipschitz hemicontractive-type mapping with Lipschitz constant $L$ such that $\Omega=F(T) \cap E P(F, A)$ is nonempty and $T p=\{p\}$ for all $p \in \Omega$. Let $x_{1}, u \in C$ be arbitrary and $\left\{x_{n}\right\}$ be a sequence generated by

$$
\left\{\begin{array}{l}
F\left(z_{n}, y\right)+\left\langle A z_{n}, y-z_{n}\right\rangle+\frac{1}{r_{n}}\left\langle y-z_{n}, z_{n}-x_{n}\right\rangle \geq 0, \forall y \in C \\
y_{n}=\left(1-\lambda_{n}\right) z_{n}+\lambda_{n} u_{n} \\
x_{n+1}=\alpha_{n} u+\beta_{n} v_{n}+\gamma_{n} z_{n}
\end{array}\right.
$$

for all $n \geq 1$, where $u_{n} \in T z_{n}, v_{n} \in T y_{n}$ such that $\left\|u_{n}-v_{n}\right\| \leq$ $2 D\left(T z_{n}, T y_{n}\right)$ and $\left\{r_{n}\right\} \subset[r, \infty)$ for some $r>0,\left\{\beta_{n}\right\},\left\{\gamma_{n}\right\} \subset[a, b]$, and $\left\{\alpha_{n}\right\} \subset(0, c)$ for some $a, b, c \in(0,1)$ satisfying the following conditions: (i) $\alpha_{n}+\beta_{n}+\gamma_{n}=1$; (ii) $\lim _{n \rightarrow \infty} \alpha_{n}=0, \sum \alpha_{n}=\infty$; (iii) $\alpha_{n}+\beta_{n} \leq \lambda_{n} \leq \lambda<\frac{1}{\sqrt{1+4 L^{2}}+1}$. Then, the sequence $\left\{x_{n}\right\}$ is bounded.

Proof. Let $p \in \Omega$. Then, $T p=\{p\}$ and $F(p, y)+\langle A p, y-p\rangle \geq 0$. Define $G(x, y)=F(x, y)+\langle A x, y-x\rangle$ for all $x, y \in C$. Then, by remark 1.4, $G$ satisfies Assumption 1.3 and $p \in E P(F, A)$ is equivalent to $G(p, y) \geq 0$ for all $y \in C$. Thus, using the definition, $z_{n}$ can be rewritten as $z_{n}=T_{r_{n}} x_{n}:=\left\{F\left(z_{n}, y\right)+\left\langle A z_{n}, y-z_{n}\right\rangle+\frac{1}{r_{n}}\left\langle y-z_{n}, z_{n}-\right.\right.$ $\left.\left.x_{n}\right\rangle \geq 0, \forall y \in C,\right\}$ and hence we have $p=T_{r_{n}} p$. From the fact that $T_{r_{n}}$ is nonexpansive, we obtain that

$$
\left\|z_{n}-p\right\|=\left\|T_{r_{n}} x_{n}-T_{r_{n}} p\right\| \leq\left\|x_{n}-p\right\| .
$$

Since $T$ is hemicontractive-type mapping and $u_{n} \in T z_{n}$, from (3.1), (3.2) and Lemma 2.1, we get

$$
\begin{aligned}
\left\|y_{n}-p\right\|^{2}= & \left\|\left(1-\lambda_{n}\right) z_{n}+\lambda_{n} u_{n}-p\right\|^{2} \\
= & \left(1-\lambda_{n}\right)\left\|z_{n}-p\right\|^{2}+\lambda_{n}\left\|u_{n}-p\right\|^{2} \\
& -\lambda_{n}\left(1-\lambda_{n}\right)\left\|z_{n}-u_{n}\right\|^{2} \\
\leq & \left(1-\lambda_{n}\right)\left\|z_{n}-p\right\|^{2}+\lambda_{n} D^{2}\left(T z_{n}, T p\right) \\
& -\lambda_{n}\left(1-\lambda_{n}\right)\left\|z_{n}-u_{n}\right\|^{2} \\
\leq & \left(1-\lambda_{n}\right)\left\|z_{n}-p\right\|^{2}+\lambda_{n}\left(\left\|z_{n}-p\right\|^{2}+\left\|z_{n}-u_{n}\right\|^{2}\right) \\
& -\lambda_{n}\left(1-\lambda_{n}\right)\left\|z_{n}-u_{n}\right\|^{2} \\
= & \left\|z_{n}-p\right\|^{2}+\lambda_{n}\left\|z_{n}-u_{n}\right\|^{2}-\lambda_{n}\left(1-\lambda_{n}\right)\left\|z_{n}-u_{n}\right\|^{2} \\
\leq & \left\|x_{n}-p\right\|^{2}+\lambda_{n}^{2}\left\|z_{n}-u_{n}\right\|^{2} .
\end{aligned}
$$

On the other hand, since $T$ is hemicontractive-type mapping and $v_{n} \in T y_{n}$, from (3.1) and (3.3), we have that

$$
\begin{aligned}
\left\|v_{n}-p\right\|^{2}= & \left(d\left(v_{n}, T p\right)\right)^{2} \leq D^{2}\left(T y_{n}, T p\right) \\
\leq & \left\|y_{n}-p\right\|^{2}+\left\|y_{n}-v_{n}\right\|^{2} \\
\leq & \left\|x_{n}-p\right\|^{2}+\lambda_{n}^{2}\left\|z_{n}-u_{n}\right\|^{2} \\
& +\left\|y_{n}-v_{n}\right\|^{2} .
\end{aligned}
$$

It follows from (3.1) that

$$
\begin{gathered}
\left\|z_{n}-y_{n}\right\|^{2}=\left\|z_{n}-\left(\left(1-\lambda_{n}\right) z_{n}+\lambda_{n} u_{n}\right)\right\|^{2} \\
=\lambda_{n}^{2}\left\|z_{n}-u_{n}\right\|^{2} .
\end{gathered}
$$

Since $T$ is $L$-Lipschitzian mapping and $\left\|u_{n}-v_{n}\right\| \leq 2 D\left(T z_{n}, T y_{n}\right)$, using (3.5) and Lemma 2.1, we get that

$$
\begin{aligned}
\left\|y_{n}-v_{n}\right\|^{2}= & \left\|\left(1-\lambda_{n}\right)\left(z_{n}-v_{n}\right)+\lambda_{n}\left(u_{n}-v_{n}\right)\right\|^{2} \\
= & \left(1-\lambda_{n}\right)\left\|z_{n}-v_{n}\right\|^{2}+\lambda_{n}\left\|u_{n}-v_{n}\right\|^{2} \\
& -\lambda_{n}\left(1-\lambda_{n}\right)\left\|z_{n}-u_{n}\right\|^{2} \\
\leq & \left(1-\lambda_{n}\right)\left\|z_{n}-v_{n}\right\|^{2}+4 \lambda_{n} D^{2}\left(T z_{n}, T y_{n}\right) \\
& -\lambda_{n}\left(1-\lambda_{n}\right)\left\|z_{n}-u_{n}\right\|^{2} \\
\leq & \left(1-\lambda_{n}\right)\left\|z_{n}-v_{n}\right\|^{2}+4 \lambda_{n} L^{2}\left\|z_{n}-y_{n}\right\|^{2} \\
& -\lambda_{n}\left(1-\lambda_{n}\right)\left\|z_{n}-u_{n}\right\|^{2} \\
= & \left(1-\lambda_{n}\right)\left\|z_{n}-v_{n}\right\|^{2}+4 \lambda_{n}^{3} L^{2}\left\|z_{n}-u_{n}\right\|^{2} \\
& -\lambda_{n}\left(1-\lambda_{n}\right)\left\|z_{n}-u_{n}\right\|^{2} \\
= & \left(1-\lambda_{n}\right)\left\|z_{n}-v_{n}\right\|^{2} \\
& +\lambda_{n}\left(4 L^{2} \lambda_{n}^{2}+\lambda_{n}-1\right)\left\|z_{n}-u_{n}\right\|^{2} .
\end{aligned}
$$

Hence, substituting (3.6) into (3.4), we obtain that

$$
\begin{aligned}
\left\|v_{n}-p\right\|^{2} \leq & \left\|x_{n}-p\right\|^{2}+\lambda_{n}^{2}\left\|z_{n}-u_{n}\right\|^{2}+\left(1-\lambda_{n}\right)\left\|z_{n}-v_{n}\right\|^{2} \\
& +\lambda_{n}\left(4 L^{2} \lambda_{n}^{2}+\lambda_{n}-1\right)\left\|z_{n}-u_{n}\right\|^{2} \\
= & \left\|x_{n}-p\right\|^{2}+\left(1-\lambda_{n}\right)\left\|z_{n}-v_{n}\right\|^{2} \\
& +\lambda_{n}\left(4 L^{2} \lambda_{n}^{2}+2 \lambda_{n}-1\right)\left\|z_{n}-u_{n}\right\|^{2}
\end{aligned}
$$


Thus, from (3.2), (3.7) and Lemma 2.1, we have that

$$
\begin{aligned}
\left\|x_{n+1}-p\right\|^{2}= & \left\|\alpha_{n} u+\beta_{n} v_{n}+\gamma_{n} z_{n}-p\right\|^{2} \\
\leq & \alpha_{n}\|u-p\|^{2}+\beta_{n}\left\|v_{n}-p\right\|^{2}+\gamma_{n}\left\|z_{n}-p\right\|^{2} \\
& -\beta_{n} \gamma_{n}\left\|z_{n}-v_{n}\right\|^{2} \\
\leq & \alpha_{n}\|u-p\|^{2} \\
& +\beta_{n}\left(\left\|x_{n}-p\right\|^{2}+\left(1-\lambda_{n}\right)\left\|z_{n}-v_{n}\right\|^{2}\right. \\
& \left.+\lambda_{n}\left(4 L^{2} \lambda_{n}^{2}+2 \lambda_{n}-1\right)\left\|z_{n}-u_{n}\right\|^{2}\right) \\
& +\gamma_{n}\left\|z_{n}-p\right\|^{2}-\beta_{n} \gamma_{n}\left\|z_{n}-v_{n}\right\|^{2} \\
= & \alpha_{n}\|u-p\|^{2}+\left(1-\alpha_{n}\right)\left\|x_{n}-p\right\|^{2} \\
& +\beta_{n}\left(1-\gamma_{n}-\lambda_{n}\right)\left\|z_{n}-v_{n}\right\|^{2} \\
& -\beta_{n} \lambda_{n}\left(1-4 L^{2} \lambda_{n}^{2}-2 \lambda_{n}\right)\left\|z_{n}-u_{n}\right\|^{2} .
\end{aligned}
$$

Then, by assumption (i), we have

$$
\begin{aligned}
\left\|x_{n+1}-p\right\|^{2} \leq & \alpha_{n}\|u-p\|^{2}+\left(1-\alpha_{n}\right)\left\|x_{n}-p\right\|^{2} \\
& -\beta_{n} \lambda_{n}\left(1-4 L^{2} \lambda_{n}^{2}-2 \lambda_{n}\right)\left\|z_{n}-u_{n}\right\|^{2} \\
& +\beta_{n}\left(\alpha_{n}+\beta_{n}-\lambda_{n}\right)\left\|z_{n}-v_{n}\right\|^{2},
\end{aligned}
$$

and from assumption (iii), we get

$$
\begin{aligned}
& 1-4 L^{2} \lambda_{n}^{2}-2 \lambda_{n} \geq 1-4 L^{2} \lambda^{2}-2 \lambda>0 \\
& \text { and }\left(\alpha_{n}+\beta_{n}\right)-\lambda_{n} \leq 0,
\end{aligned}
$$

for all $n \geq 1$. Therefore, from (3.8) and (3.9), we infer that

$$
\begin{aligned}
\left\|x_{n+1}-p\right\|^{2} & \leq \alpha_{n}\|u-p\|^{2}+\left(1-\alpha_{n}\right)\left\|x_{n}-p\right\|^{2} \\
& \leq \max \left\{\|u-p\|^{2},\left\|x_{n}-p\right\|^{2}\right\} .
\end{aligned}
$$

Thus, by induction, we have that

$$
\left\|x_{n}-p\right\|^{2} \leq \max \left\{\|u-p\|^{2},\left\|x_{1}-p\right\|^{2}\right\}
$$

which implies that $\left\{x_{n}\right\}$ is bounded. This completes the proof.

Theorem 3.2. Let $C$ be a nonempty, closed and convex subset of a real Hilbert space $H$. Let $A: C \longrightarrow H$ be a continuous monotone mapping and $F: C \times C \longrightarrow \mathbb{R}$ be a bifunction satisfying Assumption 1.3. Let $T: C \longrightarrow C B(C)$ be a Lipschitz hemicontractive-type mapping with Lipschitz constant $L$ such that $\Omega=F(T) \cap E P(F, A)$ is nonempty, closed and convex. Assume that $(I-T)$ is demiclosed at zero and $T p=\{p\}$ for all $p \in \Omega$. Let $x_{1}, u \in C$ be arbitrary and $\left\{x_{n}\right\}$ be a sequence generated by

$$
\left\{\begin{array}{l}
F\left(z_{n}, y\right)+\left\langle A z_{n}, y-z_{n}\right\rangle+\frac{1}{r_{n}}\left\langle y-z_{n}, z_{n}-x_{n}\right\rangle \geq 0, \forall y \in C \\
y_{n}=\left(1-\lambda_{n}\right) z_{n}+\lambda_{n} u_{n} \\
x_{n+1}=\alpha_{n} u+\beta_{n} v_{n}+\gamma_{n} z_{n}
\end{array}\right.
$$

for all $n \geq 1$, where $u_{n} \in T z_{n}, v_{n} \in T y_{n}$ such that $\left\|u_{n}-v_{n}\right\| \leq$ $2 D\left(T z_{n}, T y_{n}\right)$ and $\left\{r_{n}\right\} \subset[r, \infty)$ for some $r>0,\left\{\beta_{n}\right\},\left\{\gamma_{n}\right\} \subset[a, b]$, and $\left\{\alpha_{n}\right\} \subset(0, c)$ for some $a, b, c \in(0,1)$ satisfying the following conditions: (i) $\alpha_{n}+\beta_{n}+\gamma_{n}=1$; (ii) $\lim _{n \rightarrow \infty} \alpha_{n}=0, \sum \alpha_{n}=\infty$; (iii) $\alpha_{n}+\beta_{n} \leq \lambda_{n} \leq \lambda<\frac{1}{\sqrt{1+4 L^{2}}+1}$. Then, the sequence $\left\{x_{n}\right\}$ converges strongly to the point $z=P_{\Omega}(u)$.

Proof. Since $\Omega$ is nonempty, closed and convex subset of $C$, we get that $P_{\Omega}$ is well defined. Clearly, from Theorem 3.1 the sequence $\left\{x_{n}\right\}$ and hence $\left\{y_{n}\right\},\left\{z_{n}\right\}$ are bounded. As in Theorem 3.1, let $p \in \Omega$. Then, using (3.10), Lemma 2.1 and Lemma 2.2, we obtain

$$
\begin{aligned}
\left\|x_{n+1}-p\right\|^{2}= & \left\|\alpha_{n} u+\beta_{n} v_{n}+\gamma_{n} z_{n}-p\right\|^{2} \\
\leq & \left\|\beta_{n}\left(v_{n}-p\right)+\gamma_{n}\left(z_{n}-p\right)\right\|^{2} \\
& +2 \alpha_{n}\left\langle u-p, x_{n+1}-p\right\rangle \\
\leq & \beta_{n}\left\|v_{n}-p\right\|^{2}+\gamma_{n}\left\|z_{n}-p\right\|^{2}-\beta_{n} \gamma_{n}\left\|z_{n}-v_{n}\right\|^{2} \\
& +2 \alpha_{n}\left\langle u-p, x_{n+1}-p\right\rangle .
\end{aligned}
$$

Since $T_{r_{n}}$ is firmly nonexpansive, from (2.2) we have that

$$
\begin{aligned}
\left\|z_{n}-p\right\|^{2} & =\left\|T_{r_{n}} x_{n}-T_{r_{n}} p\right\|^{2} \\
& \leq\left\langle z_{n}-p, x_{n}-p\right\rangle \\
& =\frac{1}{2}\left(\left\|z_{n}-p\right\|^{2}+\left\|x_{n}-p\right\|^{2}-\left\|x_{n}-z_{n}\right\|^{2}\right)
\end{aligned}
$$

which gives that

$$
\left\|z_{n}-p\right\|^{2} \leq\left\|x_{n}-p\right\|^{2}-\left\|x_{n}-z_{n}\right\|^{2} .
$$

Thus, substituting (3.7) and (3.12) into (3.11), we obtain that

$$
\begin{aligned}
\left\|x_{n+1}-p\right\|^{2} \leq & \beta_{n}\left(\left\|x_{n}-p\right\|^{2}+\lambda_{n}\left(4 L^{2} \lambda_{n}^{2}+2 \lambda_{n}-1\right)\right. \\
& \left.\times\left\|z_{n}-u_{n}\right\|^{2}+\left(1-\lambda_{n}\right)\left\|z_{n}-v_{n}\right\|^{2}\right) \\
& +\gamma_{n}\left(\left\|x_{n}-p\right\|^{2}-\left\|x_{n}-z_{n}\right\|^{2}\right) \\
& -\beta_{n} \gamma_{n}\left\|z_{n}-v_{n}\right\|^{2}+2 \alpha_{n}\left\langle u-p, x_{n+1}-p\right\rangle \\
= & \left(1-\alpha_{n}\right)\left\|x_{n}-p\right\|^{2}-\beta_{n} \lambda_{n}\left(1-4 L^{2} \lambda_{n}^{2}-2 \lambda_{n}\right) \\
& \times\left\|z_{n}-u_{n}\right\|^{2}+\beta_{n}\left(\alpha_{n}+\beta_{n}-\lambda_{n}\right)\left\|z_{n}-v_{n}\right\|^{2} \\
& -\gamma_{n}\left\|z_{n}-x_{n}\right\|^{2}+2 \alpha_{n}\left\langle u-p, x_{n+1}-p\right\rangle .(3.13)
\end{aligned}
$$

It follows from (3.9) that

$$
\begin{aligned}
\left\|x_{n+1}-p\right\|^{2} \leq & \left(1-\alpha_{n}\right)\left\|x_{n}-p\right\|^{2} \\
& +2 \alpha_{n}\left\langle u-p, x_{n+1}-p\right\rangle .
\end{aligned}
$$

Next, we consider two cases:

Case 1. Suppose that there exists $n_{0} \in \mathbb{N}$ such that $\left\{\left\|x_{n}-p\right\|\right\}$ is decreasing for all $n \geq n_{0}$. Then, we get that, $\left\{\left\|x_{n}-p\right\|\right\}$ is convergent. Thus, from (3.13) and (3.9), we have that

$$
\begin{aligned}
\beta_{n} \lambda_{n}\left(1-4 L^{2} \lambda_{n}^{2}-2 \lambda_{n}\right)\left\|z_{n}-u_{n}\right\|^{2} \leq & \left(1-\alpha_{n}\right)\left\|x_{n}-p\right\|^{2} \\
& -\left\|x_{n+1}-p\right\|^{2} \\
& +2 \alpha_{n}\left\langle u-p, x_{n+1}-p\right\rangle .
\end{aligned}
$$

Hence, from (3.9) and the fact that $\alpha_{n} \rightarrow 0$ as $n \rightarrow \infty$, we have that

$$
\left\|z_{n}-u_{n}\right\| \rightarrow 0 \text { as } n \rightarrow \infty,
$$

which implies that

$$
d\left(z_{n}, T z_{n}\right) \leq\left\|z_{n}-u_{n}\right\| \rightarrow 0 \text { as } n \rightarrow \infty .
$$

In addition, from (3.9) and (3.13), we find that

$$
\begin{aligned}
\gamma_{n}\left\|z_{n}-x_{n}\right\|^{2} \leq & \left(1-\alpha_{n}\right)\left\|x_{n}-p\right\|^{2}-\left\|x_{n+1}-p\right\|^{2} \\
& +2 \alpha_{n}\left\langle u-p, x_{n+1}-p\right\rangle .
\end{aligned}
$$

Thus, since $\alpha_{n} \rightarrow 0$ as $n \rightarrow \infty$, it follows from the assumption of $\left\{\gamma_{n}\right\}$ that

$$
\lim _{n \rightarrow \infty}\left\|z_{n}-x_{n}\right\|=0
$$

And using the Lipschitz continuity of $T$, (3.5) and (3.15), we get that

$$
\begin{aligned}
\left\|z_{n}-v_{n}\right\| & \leq\left\|z_{n}-u_{n}\right\|+\left\|u_{n}-v_{n}\right\| \\
& \leq\left\|z_{n}-u_{n}\right\|+2 L\left\|z_{n}-y_{n}\right\| \\
& =\left\|z_{n}-u_{n}\right\|+2 L \lambda_{n}\left\|z_{n}-u_{n}\right\| \rightarrow 0 \text { as } n \rightarrow \infty .
\end{aligned}
$$

Therefore, from (3.17), (3.18), definition of $\left\{x_{n+1}\right\}$ and the fact that $\alpha_{n} \rightarrow 0$ as $n \rightarrow \infty$, we get

$$
\begin{aligned}
\left\|x_{n+1}-x_{n}\right\| & \leq\left\|x_{n+1}-z_{n}\right\|+\left\|z_{n}-x_{n}\right\| \\
& =\left\|\alpha_{n}\left(u-z_{n}\right)+\beta_{n}\left(v_{n}-z_{n}\right)\right\|+\left\|z_{n}-x_{n}\right\| \\
& \leq \alpha_{n}\left\|u-z_{n}\right\|+\beta_{n}\left\|v_{n}-z_{n}\right\|+\left\|z_{n}-x_{n}\right\| \rightarrow 0 \text { as } n \rightarrow \infty .
\end{aligned}
$$


Now, let $z=P_{\Omega}(u)$. We claim that $\limsup _{n \rightarrow \infty}\left\langle u-z, x_{n+1}-z\right\rangle \leq 0$. Since $\left\{x_{n+1}\right\}$ is a bounded sequence in a real Hilbert space $H$, which is reflexive Banach space, there exists a subsequence $\left\{x_{n_{i}+1}\right\}$ of $\left\{x_{n+1}\right\}$ and an element in $H$, say $q$, such that

$$
x_{n_{i}+1} \rightarrow q \text { and } \limsup _{n \rightarrow \infty}\left\langle u-z, x_{n+1}-z\right\rangle=\lim _{i \rightarrow \infty}\left\langle u-z, x_{n_{i}+1}-z\right\rangle .
$$

Since, $C$ is weakly closed, we have $q \in C$ and from (3.19) it follows that $x_{n_{i}} \rightarrow q$ as $i \rightarrow \infty$. From (3.17), we obtain that $z_{n_{i}} \rightarrow q$ as $i \rightarrow \infty$ and hence using the assumption that $(I-T)$ is demiclosed at zero and (3.16), we conclude that

$$
q \in F(T) .
$$

In addition, from (3.1) and replacing $n$ by $n_{i}$ in (3.17), we have that

$$
\left\|x_{n_{i}}-T_{r_{n_{i}}} x_{n_{i}}\right\|=\left\|x_{n_{i}}-z_{n_{i}}\right\| \rightarrow 0 \text { as } i \rightarrow \infty .
$$

Thus, since $T_{r_{n_{i}}}$ is nonexpansive, then $\left(I-T_{r_{n_{i}}}\right)$ is demiclosed at zero and hence since $x_{n_{i}} \rightarrow q$ as $i \rightarrow \infty$, we obtain that $q=T_{r_{n_{i}}} q$, that is, $F(q, y)+\langle A q, y-q\rangle \geq 0$ for all $y \in C$. Hence, we have

$$
q \in E P(F, A),
$$

which implies that $q \in \Omega$. Hence, since $z=P_{\Omega}(u)$ and $x_{n_{i}} \rightarrow q$, from (2.1) we get that

$$
\begin{aligned}
\limsup _{n \rightarrow \infty}\left\langle u-z, x_{n+1}-z\right\rangle & =\lim _{i \rightarrow \infty}\left\langle u-z, x_{n_{i}+1}-z\right\rangle \\
& =\langle u-z, q-z\rangle \leq 0
\end{aligned}
$$

Thus, since $z \in \Omega$, from (3.14), (3.20), assumptions of $\left\{\alpha_{n}\right\}$ and Lemma 2.5, we get that

$$
\left\|x_{n}-z\right\| \rightarrow 0 \text { as } n \rightarrow \infty .
$$

Hence, $x_{n} \rightarrow z=P_{\Omega}(u)$.

Case 2. Suppose that there exists a subsequence $\left\{n_{j}\right\}$ of $\{n\}$ such that

$$
\left\|x_{n_{j}}-p\right\|<\left\|x_{n_{j}+1}-p\right\|,
$$

for all $j \in \mathbb{N}$. Then, by Lemma 2.6 , there exist a nondecreasing sequence $\left\{m_{k}\right\} \subset \mathbb{N}$ such that $m_{k} \rightarrow \infty$, and

$$
\left\|x_{m_{k}}-p\right\| \leq\left\|x_{m_{k}+1}-p\right\| \text { and }\left\|x_{k}-p\right\| \leq\left\|x_{m_{k}+1}-p\right\|,
$$

for all $k \in \mathbb{N}$. Thus, from (3.13), (3.9) and the fact that $\alpha_{n} \rightarrow 0$, we get that

$$
\left\|z_{m_{k}}-u_{m_{k}}\right\| \rightarrow 0 \text { and }\left\|z_{m_{k}}-x_{m_{k}}\right\| \rightarrow 0 \text { as } k \rightarrow \infty .
$$

Hence, since $z=P_{\Omega}(u)$, following the method in Case 1, we obtain that

$$
\limsup _{k \rightarrow \infty}\left\langle u-z, x_{m_{k}+1}-z\right\rangle \leq 0 .
$$

Now, Because $z \in \Omega$, from (3.14), we have that

$$
\begin{aligned}
\left\|x_{m_{k}+1}-z\right\|^{2} \leq & \left(1-\alpha_{m_{k}}\right)\left\|x_{m_{k}}-z\right\|^{2} \\
& +2 \alpha_{m_{k}}\left\langle u-z, x_{m_{k}+1}-z\right\rangle,
\end{aligned}
$$

and hence, since $z \in \Omega,(3.21)$ and (3.23) imply that

$$
\begin{aligned}
\alpha_{m_{k}}\left\|x_{m_{k}}-z\right\|^{2} \leq & \left\|x_{m_{k}}-z\right\|^{2}-\left\|x_{m_{k}+1}-z\right\|^{2} \\
& +2 \alpha_{m_{k}}\left\langle u-z, x_{m_{k}+1}-z\right\rangle \\
\leq & 2 \alpha_{m_{k}}\left\langle u-z, x_{m_{k}+1}-z\right\rangle .
\end{aligned}
$$

Hence, the fact that $\alpha_{m_{k}}>0$ imply that

$$
\left\|x_{m_{k}}-z\right\|^{2} \leq 2\left\langle u-z, x_{m_{k}+1}-z\right\rangle .
$$

Thus, using (3.22) we get that $\left\|x_{m_{k}}-z\right\| \rightarrow 0$ as $k \rightarrow \infty$. This together with (3.23) and (3.22) implies that $\left\|x_{m_{k}+1}-z\right\| \rightarrow 0$ as $k \rightarrow \infty$. Since $z \in \Omega$, we obtain $\left\|x_{k}-z\right\| \leq\left\|x_{m_{k}+1}-z\right\|$, for all $k \in \mathbb{N}$. Thus, we get that $x_{k} \rightarrow z$ as $k \rightarrow \infty$. Consequently, from both the above cases, we deduce that $\left\{x_{n}\right\}$ converges strongly to $z=P_{\Omega}(u)$. This completes the proof.
As a direct consequence of our main result, we obtain the following results:

Corollary 3.3. Let $C$ be a nonempty, closed and convex subset of a real Hilbert space $H$. Let $A: C \longrightarrow H$ be a continuous monotone mapping and $F: C \times C \longrightarrow \mathbb{R}$ be a bifunction satisfying Assumption 1.3. Let $T: C \longrightarrow C B(C)$ be a Lipschitz pseudocontractive mapping with Lipschitz constant $L$ such that $\Omega=F(T) \cap E P(F, A)$ is nonempty, closed and convex. Assume that $(I-T)$ is demiclosed at zero and $T p=\{p\}$ for all $p \in F(T)$. Let $x_{1}, u \in C$ be arbitrary and $\left\{x_{n}\right\}$ be a sequence generated by

$\left\{\begin{array}{l}F\left(z_{n}, y\right)+\left\langle A z_{n}, y-z_{n}\right\rangle+\frac{1}{r_{n}}\left\langle y-z_{n}, z_{n}-x_{n}\right\rangle \geq 0, \forall y \in C, \\ y_{n}=\left(1-\lambda_{n}\right) z_{n}+\lambda_{n} u_{n}, \\ x_{n+1}=\alpha_{n} u+\beta_{n} v_{n}+\gamma_{n} z_{n},\end{array}\right.$

for all $n \geq 1$, where $u_{n} \in T z_{n}, v_{n} \in T y_{n}$ such that $\left\|u_{n}-v_{n}\right\| \leq$ $2 D\left(T z_{n}, T y_{n}\right)$ and $\left\{r_{n}\right\} \subset[r, \infty)$ for some $r>0,\left\{\beta_{n}\right\},\left\{\gamma_{n}\right\} \subset[a, b]$, and $\left\{\alpha_{n}\right\} \subset(0, c)$ for some $a, b, c \in(0,1)$ satisfying the following conditions: (i) $\alpha_{n}+\beta_{n}+\gamma_{n}=1$; (ii) $\lim _{n \rightarrow \infty} \alpha_{n}=0, \sum \alpha_{n}=\infty$; (iii) $\alpha_{n}+\beta_{n} \leq \lambda_{n} \leq \lambda<\frac{1}{\sqrt{1+4 L^{2}}+1}$. Then, the sequence $\left\{x_{n}\right\}$ converges strongly to the point $z=P_{\Omega}(u)$.

Proof. Since a Lipschitz pseudocontractive multi-valued mapping $T$ with $F(T) \neq \emptyset$ and $T(p)=\{p\}, \forall p \in F(T)$ is Lipschitz hemicontractive-type, we obtain that the desired result from Theorem 3.2. This completes the proof.

If, in Theorem 3.2, we assume that $A \equiv 0$, then we obtain the following corollary:

Corollary 3.4. Let $C$ be a nonempty, closed and convex subset of a real Hilbert space $H$. Let $F: C \times C \longrightarrow \mathbb{R}$ be a bifunction satisfying Assumption 1.3 and $T: C \longrightarrow C B(C)$ be a Lipschitz hemicontractivetype mapping with Lipschitz constant $L$ such that $\Omega=F(T) \cap E P(F)$ is nonempty, closed and convex. Assume that $(I-T)$ is demiclosed at zero and $T p=\{p\}$ for all $p \in \Omega$. Let $x_{1}, u \in C$ be arbitrary and $\left\{x_{n}\right\}$ be a sequence generated by

$$
\left\{\begin{array}{l}
F\left(z_{n}, y\right)+\frac{1}{r_{n}}\left\langle y-z_{n}, z_{n}-x_{n}\right\rangle \geq 0, \forall y \in C, \\
y_{n}=\left(1-\lambda_{n}\right) z_{n}+\lambda_{n} u_{n} \\
x_{n+1}=\alpha_{n} u+\beta_{n} v_{n}+\gamma_{n} z_{n},
\end{array}\right.
$$

for all $n \geq 1$, where $u_{n} \in T z_{n}, v_{n} \in T y_{n}$ such that $\left\|u_{n}-v_{n}\right\| \leq$ $2 D\left(T z_{n}, T y_{n}\right)$ and $\left\{r_{n}\right\} \subset[r, \infty)$ for some $r>0,\left\{\beta_{n}\right\},\left\{\gamma_{n}\right\} \subset[a, b]$, and $\left\{\alpha_{n}\right\} \subset(0, c)$ for some $a, b, c \in(0,1)$ satisfying the following conditions: (i) $\alpha_{n}+\beta_{n}+\gamma_{n}=1$; (ii) $\lim _{n \rightarrow \infty} \alpha_{n}=0, \sum \alpha_{n}=\infty$; (iii) $\alpha_{n}+\beta_{n} \leq \lambda_{n} \leq \lambda<\frac{1}{\sqrt{1+4 L^{2}}+1}$. Then, the sequence $\left\{x_{n}\right\}$ converges strongly to the point $z=P_{\Omega}(u)$.

If, in Theorem 3.2, we assume that $F(x, y)=0$ for all $x, y \in C$, then we obtain the following corollary.

Corollary 3.5. Let $C$ be a nonempty, closed and convex subset of a real Hilbert space $H$. Let $A: C \longrightarrow H$ be a continuous monotone mapping and $T: C \longrightarrow C B(C)$ be a Lipschitz hemicontractive-type mapping with Lipschitz constant $L$ such that $\Omega=F(T) \cap V I(C, A)$ is nonempty, closed and convex. Assume that $(I-T)$ is demiclosed at zero and $T p=\{p\}$ for all $p \in \Omega$. Let $x_{1}, u \in C$ be arbitrary and $\left\{x_{n}\right\}$ be a sequence generated by

$$
\left\{\begin{array}{l}
\left\langle A z_{n}, y-z_{n}\right\rangle+\frac{1}{r_{n}}\left\langle y-z_{n}, z_{n}-x_{n}\right\rangle \geq 0, \forall y \in C, \\
y_{n}=\left(1-\lambda_{n}\right) z_{n}+\lambda_{n} u_{n} \\
x_{n+1}=\alpha_{n} u+\beta_{n} v_{n}+\gamma_{n} z_{n}
\end{array}\right.
$$

for all $n \geq 1$, where $u_{n} \in T z_{n}, v_{n} \in T y_{n}$ such that $\left\|u_{n}-v_{n}\right\| \leq$ $2 D\left(T z_{n}, T y_{n}\right)$ and $\left\{r_{n}\right\} \subset[r, \infty)$ for some $r>0,\left\{\beta_{n}\right\},\left\{\gamma_{n}\right\} \subset[a, b]$, and $\left\{\alpha_{n}\right\} \subset(0, c)$ for some $a, b, c \in(0,1)$ satisfying the following conditions: (i) $\alpha_{n}+\beta_{n}+\gamma_{n}=1$; (ii) $\lim _{n \rightarrow \infty} \alpha_{n}=0, \sum \alpha_{n}=\infty$; (iii) $\alpha_{n}+\beta_{n} \leq \lambda_{n} \leq \lambda<\frac{1}{\sqrt{1+4 L^{2}}+1}$. Then, the sequence $\left\{x_{n}\right\}$ converges strongly to the point $z=P_{\Omega}(u)$. 
If, in Theorem 3.2, we assume that $T$ is a single-valued hemicontractive mapping from $C$ into itself, then we obtain the following corollary.

Corollary 3.6. Let $C$ be a nonempty, closed and convex subset of a real Hilbert space $H$. Let $A: C \longrightarrow H$ be a continuous monotone mapping and $F: C \times C \longrightarrow \mathbb{R}$ be a bifunction satisfying Assumption 1.3. Let $T: C \longrightarrow C$ be a Lipschitz hemicontractive mapping with Lipschitz constant $L$ such that $\Omega=F(T) \cap E P(F, A)$ is nonempty, closed and convex. Assume that $(I-T)$ is demiclosed at zero. Let $x_{1}, u \in C$ be arbitrary and $\left\{x_{n}\right\}$ be a sequence generated by

$$
\left\{\begin{array}{l}
F\left(z_{n}, y\right)+\left\langle A z_{n}, y-z_{n}\right\rangle+\frac{1}{r_{n}}\left\langle y-z_{n}, z_{n}-x_{n}\right\rangle \geq 0, \forall y \in C, \\
y_{n}=\left(1-\lambda_{n}\right) z_{n}+\lambda_{n} T z_{n} \\
x_{n+1}=\alpha_{n} u+\beta_{n} T y_{n}+\gamma_{n} z_{n}
\end{array}\right.
$$

for all $n \geq 1$, where $\left\{r_{n}\right\} \subset[r, \infty)$ for some $r>0,\left\{\beta_{n}\right\},\left\{\gamma_{n}\right\} \subset[a, b]$, and $\left\{\alpha_{n}\right\} \subset(0, c)$ for some $a, b, c \in(0,1)$ satisfying the following conditions: (i) $\alpha_{n}+\beta_{n}+\gamma_{n}=1$; (ii) $\lim _{n \rightarrow \infty} \alpha_{n}=0, \sum \alpha_{n}=\infty$; (iii) $\alpha_{n}+\beta_{n} \leq \lambda_{n} \leq \lambda<\frac{1}{\sqrt{1+L^{2}}+1}$. Then, the sequence $\left\{x_{n}\right\}$ converges strongly to the point $z=P_{\Omega}(u)$.

If, in Theorem 3.2, we assume that $T=I$, where $I$ is the identity mapping on $C$, then we obtain the following corollary.

Corollary 3.7. Let $C$ be a nonempty, closed and convex subset of a real Hilbert space $H$. Let $A: C \longrightarrow H$ be a continuous monotone mapping and $F: C \times C \longrightarrow \mathbb{R}$ be a bifunction satisfying Assumption 1.3 such that $\operatorname{EP}(F, A)$ is nonempty. Let $x_{1}, u \in C$ be arbitrary and $\left\{x_{n}\right\}$ be a sequence generated by

$$
\left\{\begin{array}{l}
F\left(z_{n}, y\right)+\left\langle A z_{n}, y-z_{n}\right\rangle+\frac{1}{r_{n}}\left\langle y-z_{n}, z_{n}-x_{n}\right\rangle \geq 0, \forall y \in C, \\
x_{n+1}=\alpha_{n} u+\left(1-\alpha_{n}\right) z_{n},
\end{array}\right.
$$

for all $n \geq 1$, where $\left\{r_{n}\right\} \subset[r, \infty)$ for some $r>0$ and $\left\{\alpha_{n}\right\} \subset(0, c)$ for some $c \in(0,1)$ such that $\lim _{n \rightarrow \infty} \alpha_{n}=0$ and $\sum \alpha_{n}=\infty$. Then, the sequence $\left\{x_{n}\right\}$ converges strongly to the point $z=P_{E P(F, A)}(u)$.

Remark 3.8. Theorem 3.2 extends the results of Iiduka and Takahashi [7], Meche et al.[10], Takahashi and Takahashi [16, 17], Wang et al.[18], Wong et al.[4] in the sense that our algorithm scheme provides strong convergence to a common element of the set of solutions of a generalized equilibrium problem for continuous monotone mapping and the set of fixed points of a Lipschitz hemicontractive-type multi-valued mapping. We have used the demiclosedness principle to show that $q \in \operatorname{EP}(F, A)$ in Theorem 3.2, which is a little simpler than using Assumption 1.3.

\section{Acknowledgement}

This work was completed with the partial support of Simons Foundation based in Botswana International University of Science and Technology.

\section{References}

[1] R.P. Agrawal, D. O'Regan, D.R. Sahu, "Fixed Point Theory for Lipschitzian-type Mappings with Applications," Springer, New York, 2009.

[2] V. Berinde, M. Păcurar, "The role of Pompeiu-Hausdorff metric in fixed point theory," Creat. Math. Inform., 22 (2) (2013) 143-150.

[3] E. Blum, W. Oettli, "From optimization and variational inequalities to equilibrium problems," Math. Stud., 63 (1994) 123-145.

[4] L. Ceng, A. Petrusel, M. Wong, "Strong convergence theorem for a generalized equilibrium problem and a pseudocontractive mapping in a Hilbert space," Taiwan J. Math., 14 (2010) 1881-1901.

[5] C.E. Chidume, C.O. Chidume, N. Djitte, M.S. Minjibir, "Convergence theorems for fixed points of multi-valued strictly pseudocontractive mapping in Hilbert spaces," Abstr. Appl. Anal., 2013, Article ID 629468 10 pages. http://dx.doi.org/10.1155/2013/629468.

[6] P. L. Combettes, S. A. Hirstoaga, "Equilibrium programming in Hilbert spaces," J. Nonlinear Convex Anal., 6 (2005) 117-136.
[7] H. Iiduka, W. Takahashi, "Strong convergence theorems for nonexpansive mappings and inverse-strongly monotone mappings," Nonlinear Analysis, 61 (2005) 341-350.

[8] F.O. Isiogugu, M.O. Osilike, "convergence theorems for new classes of multi-valued hemicontractive-type mappings," Fixed point theory Appl., 2014 (2014):93, 12 pages.

[9] P. E. Mainge, "Strong convergence of projected subgradient methods for nonsmooth and nonstrictly convex minimization," Set-Valued Anal. 16 (2008) 899-912.

[10] T. H. Meche, M. G. Sangago, H. Zegeye, "Iterative methods for a fixed point of hemicontractive-type mapping and a solution of a variational inequality problem," Creat. Math. Inform., 25 (2) (2016) 183-196.

[11] A. Moudafi, "Weak convergence theorems for nonexpansive mapping and equilibrium," J. Nonlinear Convex Anal., 9(1) (2008), 37-43.

[12] S. B. Nadler, Jr., "Multi-valued contraction mappings," Pacific J. Math., 30 (1969) 475-487.

[13] A. Rafiq, "On Mann iteration in Hilbert spaces," Nonlinear Analysis, 66 (2007) 2230-2236

[14] K.P.R. Sastry, G.V.R. Babu, "Convergence of Ishikawa iterates for a multi-valued mapping with a fixed point," Czechoslovak Math. J., 55 (130) (2005) 817-826.

[15] N. Shahzad, H. Zegeye, "On Mann and Ishikawa iteration schemes for multi-valued maps in Banach spaces," Nonlinear Analysis: Theory Method and Application, 71 (2009) 838-844.

[16] S. Takahashi, W. Takahashi, "Viscosity approximation methods for equilibrium problems and fixed point problems in Hilbert spaces," $J$. Math. Anal. Appl., 331 (2007) 506-515.

[17] S. Takahashi, W. Takahashi, "Strong convergence theorem for a generalized equilibrium problem and nonexpansive mapping in a Hilbert space," Nonlinear Anal., 69 (2008) 1025-1033.

[18] S. Wang, H. Zhou, J. Song, "Viscosity approximation methods for equilibrium problems and fixed point problems of nonexpansive mappings and inverse-strongly monotone mappings," Method Appl. Anal., 14 (4) (2007) 405-420.

[19] S. T. Woldeamanuel, M. G. Sangago, H. Zegeye, "Strong convergence theorems for a common fixed point of a finite family of Lipchitz hemicontractive-type multi-valued mappings," Adv. Fixed Point Theory, 5 (2) (2015) 228-253.

[20] H. K. Xu, "Another control condition in an iterative method for nonexpansive mappings," Bull. Aust. Math. Soc., 65 (2002) 109-113.

[21] Y. Yu, Z. Wu, P. Yang, "An iterative algorithm for hemi-contractive mappings in Banach spaces," Abstr. Appl. Anal., 2012, Article ID 264103, 11 pages. doi: 10.1155/2012/264103.

[22] H. Zegeye, N. Shahzad, "Convergence of Manns type iteration method for generalized asymptotically nonexpansive mappings," Comput. Math. Appl., 62 (2011) 4007-4014. 